\title{
Using bisphenol-A to study the onset of polycystic ovarian syndrome
}

\author{
Ulrike Klenke $^{1+*}$ and B. lan Hutchins ${ }^{1,2+*}$ \\ Cellular and Developmental Neurobiology Section, National Institute of Neurological Disorders and Stroke, National Institutes of Health, Bethesda, MD, USA \\ 2 Pharmacology Research Associate Program, National Institute of General Medical Sciences, National Institutes of Health, Bethesda, MD, USA \\ *Correspondence: klenkee@ninds.nih.gov; bruce.hutchins@nih.gov \\ +Ulrike Klenke and B. lan Hutchins have contributed equally to this work.
}

\section{A commentary on}

Neonatal exposure to bisphenol a and reproductive and endocrine alterations resembling the polycystic ovarian syndrome in adult rats

by Fernandez, M., Bourguignon, N., LuxLantos, V., and Libertun, C. (2010). Environ. Health Perspect. 118, 1217-1222.

For over 30 years, scientists have focused on the impact of endocrine disrupting chemicals (EDC) on human health. These synthetic or natural compounds can mimic and/or antagonize the action of endogenous hormones. Exposure to EDCs has been linked to reduced fertility, onset of diseases like diabetes, endometriosis, and some cancers (Diamanti-Kandarakis et al., 2009). However, exactly how EDCs cause these effects is not clear. Many of the observed effects can appear throughout life, even though exposure might have occurred only shortly during early development. Exposure dose has a tremendous effect, with very low doses causing adverse effects even though being well below the determined "no effect" levels (DiamantiKandarakis et al., 2009).

One of the investigated EDCs is Bisphenol-A (BPA), a chemical produced in large quantities for production of polycarbonate plastics and epoxy resin. BPA has adverse effects on fertility, development, and behavior in lab animals (Richter et al., 2007). Furthermore, a recent study showed that BPA levels were significantly higher in women with polycystic ovarian syndrome (PCOS) compared to normal women and could possibly be one of many underlying causes of this disorder (Kandaraki et al., 2011).

Polycystic ovarian syndrome is a common endocrine disorder in women of reproductive age, characterized by ovulatory and menstrual dysfunction, hyperan- drogenism (e.g., hirsutism), and polycystic ovaries as well as metabolic symptoms, e.g., insulin resistance and obesity (Barontini et al., 2001). However, the primary defects in the hypothalamus-pituitary-ovary axis underlying this disorder have not been clearly identified. Women with PCOS show an acceleration of the GnRH pulse generator activity. This causes inappropriate gonadotropin synthesis and release with elevated LH and low FSH levels, resulting in excess production of androgens (Barontini et al., 2001). The heterogeneity of this disorder together with the absence of good animal models has caused tremendous problems studying this syndrome in the laboratory. A recent study (Fernandez et al., 2010) introduces a new animal model of PCOS that addresses shortcomings of existing models and may help dissect the molecular causes of this syndrome.

Abnormally high androgen levels may contribute to PCOS, potentially reducing natural negative feedback on GnRH release and LH levels. Fernandez et al. (2010) therefore quantified the change in sex hormone levels in adulthood caused by neonatal exposure to BPA. Rats given subcutaneous injections daily of 50 or $500 \mu \mathrm{g} /$ day from postnatal days 1-10 had higher levels of circulating testosterone and estrogen and lower levels of progesterone as adults. Androgen and serum BPA levels are both elevated in women with PCOS (Eagleson et al., 2000). However, previous studies were unable to discern whether BPA raised testosterone levels or whether elevated testosterone levels prevented clearance of BPA. These new results suggest that juvenile exposure to BPA can cause increased androgen levels in adulthood.

$\mathrm{GnRH}$ release frequency increases upon neonatal administration of BPA (Fernandez et al., 2009). This could be the result of impaired ovarian functionality. Therefore, Fernandez et al. (2010) examined ovarian morphology. BPA-treated animals (either 50 or $500 \mu \mathrm{g} /$ day) had smaller ovaries (although body weight was unaffected) and lower numbers of antral follicles. Animals treated with $500 \mu \mathrm{g} /$ day BPA as neonates also had ovarian cysts, a phenomenon shared with other animal models of PCOS (Sotomayor-Zarate et al., 2008).

Since ovarian morphology and circulating sex hormone levels are altered, is fertility also impaired by neonatal exposure to $\mathrm{BPA}$ ? To address this question, oocytes were counted in the ampulla of the oviduct on the morning of estrus. Strikingly, animals that had been treated with $500 \mu \mathrm{g} /$ day BPA as neonates did not ovulate; however, those treated with 50 or $5 \mu \mathrm{g} /$ day BPA presented the same number of oocytes as controls. How did these treatments affect overall fertility? Unsurprisingly, animals that had been exposed to $500 \mu \mathrm{g} /$ day BPA did not deliver any pups. However, those animals treated with $50 \mu \mathrm{g} /$ day BPA delivered $\sim 30 \%$ fewer pups, indicating an effect on fertility independent of reduced numbers of oocytes. These results suggest that juvenile exposure to BPA can have lasting effects on the mammalian reproductive cycle throughout adulthood.

This work introduces a new way to induce PCOS-like traits in a rodent model. Yet, metabolic malfunctions also occur in clinical PCOS; these were not investigated. In fact, while human PCOS is associated with increased risk for obesity, these BPA-treated animals showed no difference in weight. Thus, this new model of PCOS may be best suited for studying the mechanisms of onset of the androgen feedback cascade that is hypothesized to initiate PCOS. Rising androgen levels during puberty were postulated to reduce negative progesterone feedback on $\mathrm{GnRH}$ pulsatility and initiate the adult pattern of GnRH release (Blank et al., 2007). An early rise in androgen levels could therefore 
initiate this process early, tipping hormone production in favor of a positive feedback cascade of progressive GnRH release and androgen production (Tsutsumi and Webster, 2009). Current animal models bypass the onset of androgen imbalances by their direct introduction. With a better understanding of the onset of PCOS, it may be possible to develop early treatments that interrupt the development of this syndrome.

\section{ACKNOWLEDGMENTS}

The authors are supported by the Intramural Research Program of the National Institute of Neurological Disorders and Stroke, National Institutes of Health (Ulrike Klenke and B. Ian Hutchins) and a fellowship from the Pharmacology Research Associate (PRAT) Program, National Institute of General Medical Sciences, National Institutes of Health (B. Ian Hutchins).

\section{REFERENCES}

Barontini, M., Garcia-Rudaz, M. C., and Veldhuis, J. D. (2001). Mechanisms of hypothalamic-pituitary- gonadal disruption in polycystic ovarian syndrome. Arch. Med. Res. 32, 544-552.

Blank, S. K., McCartney, C. R., Helm, K. D., and Marshall, J. C. (2007). Neuroendocrine effects of androgens in adult polycystic ovary syndrome and female puberty. Semin. Reprod. Med. 25, 352-359.

Diamanti-Kandarakis, E., Bourguignon, J. P., Giudice, L. C., Hauser, R., Prins, G. S., Soto, A. M., Zoeller, R. T., and Gore, A. C. (2009). Endocrine-disrupting chemicals: an Endocrine Society scientific statement. Endocr. Rev. 30, 293-342.

Eagleson, C. A., Gingrich, M. B., Pastor, C. L., Arora, T. K., Burt, C. M., Evans, W. S., and Marshall, J. C. (2000). Polycystic ovarian syndrome: evidence that flutamide restores sensitivity of the gonadotropinreleasing hormone pulse generator to inhibition by estradiol and progesterone. J. Clin. Endocrinol. Metab. 85, 4047-4052.

Fernandez, M., Bianchi, M., Lux-Lantos, V., and Libertun, C. (2009). Neonatal exposure to bisphenol a alters reproductive parameters and gonadotropin releasing hormone signaling in female rats. Environ. Health Perspect. 117, 757-762.

Fernandez, M., Bourguignon, N., Lux-Lantos, V., and Libertun, C. (2010). Neonatal exposure to bisphenol a and reproductive and endocrine alterations resembling the polycystic ovarian syndrome in adult rats. Environ. Health Perspect. 118, 1217-1222.

Kandaraki, E., Chatzigeorgiou, A., Livadas, S., Palioura, E., Economou, F., Koutsilieris, M., Palimeri, S., Panidis, D., and Diamanti-Kandarakis, E. (2011).
Endocrine disruptors and polycystic ovary syndrome (PCOS): elevated serum levels of bisphenol A in women with PCOS. J. Clin. Endocrinol. Metab. 96, E480-E484.

Richter, C. A., Birnbaum, L. S., Farabollini, F., Newbold, R. R., Rubin, B. S., Talsness, C. E., Vandenbergh, J. G., Walser-Kuntz, D. R., and vom Saal, F. S. (2007). In vivo effects of bisphenol A in laboratory rodent studies. Reprod. Toxicol. 24, 199-224.

Sotomayor-Zarate, R., Dorfman, M., Paredes, A., and Lara, H. E. (2008). Neonatal exposure to estradiol valerate programs ovarian sympathetic innervation and follicular development in the adult rat. Biol. Reprod. 78, 673-680.

Tsutsumi, R., and Webster, N. J. (2009). GnRH pulsatility, the pituitary response and reproductive dysfunction. Endocr. J. 56, 729-737.

Received: 31 March 2011; accepted:29April 2011; published online: 10 May 2011.

Citation: Klenke U and Hutchins BI (2011) Using bisphenol-A to study the onset of polycystic ovarian syndrome. Front. Endocrin. 2:12. doi: 10.3389/fendo.2011.00012

This article was submitted to Frontiers in Neuroendocrine Science, a specialty of Frontiers in Endocrinology.

Copyright (C) 2011 Klenke and Hutchins. This is an openaccess article subject to a non-exclusive license between the authors and Frontiers Media SA, which permits use, distribution and reproduction in other forums, provided the original authors and source are credited and other Frontiers conditions are complied with. 